\title{
Catalogue of the bryophytes of Sulawesi. Supplement 1: new species records
}

\author{
N.S. Ariyanti ${ }^{1}$, S.R. Gradstein², S.G. Sporn², R. Angelika ${ }^{3}$, B.C. Tan ${ }^{4}$
}

Key words

bryophyte flora

Indonesia

Sulawesi
Abstract We report 177 bryophyte species (61 mosses, 115 liverworts, 1 hornwort) new to Sulawesi, raising the total number of bryophytes species recorded from the island to 653. The new combination Chiloscyphus morobeanus (Piippo) Gradst. comb. nov. is made.

Published on 30 October 2009

\section{INTRODUCTION}

Gradstein et al. (2005) published a first catalogue of the bryophytes of Sulawesi and listed 476 species, including 340 mosses (Bryophyta s.str.), 134 liverworts (Marchantiophyta) and 2 hornworts (Anthocerotophyta). These numbers were significantly lower than those recorded from other major Malesian islands and suggested that Sulawesi is less well collected than the other islands.

Since the appearance of the catalogue, considerable additional collecting was done in Central Sulawesi, in Lore Lindu National Park and surrounding areas in the framework of the 'STORMA' project of the universities of Palu (Sulawesi), Bogor (Java) and Göttingen (Germany) funded by the German Research Foundation (e.g., Tscharntke et al. 2007; www.storma.de). Some new liverwort records based on these collections were published by Ariyanti \& Gradstein (2007). In addition, new bryophyte reports for Sulawesi were published by Eggers (2006).

In this paper we present a synthesis of the species of bryophytes recorded as new to Sulawesi since the appearance of the catalogue. The list (Appendix) includes 177 species (61 mosses, 115 liverworts, 1 hornwort), raising the total number of bryophyte species recorded from Sulawesi to 653 . Especially noteworthy is the large number of liverworts new to Sulawesi, which almost doubles the total number of liverwort species recorded (from 134 to 249). Vouchers of the new records are kept in BIOT, BO, CEB and/or GOET unless otherwise indicated.

The new figures indicate that Sulawesi has about half the number of bryophyte species recorded from the Philippines and Borneo, and about $1 / 3$ that of New Guinea. Only five bryophyte species or less than $1 \%$ of the total bryophyte flora are endemic to Sulawesi (Gradstein et al. 2005) but the island has a relatively high concentration of eastern Malesian liverwort species, about seven times more than western ones. The high number of eastern Malesian taxa is in support of the Wallace Line (Ariyanti \& Gradstein 2007).

\footnotetext{
${ }^{1}$ Department of Biology, Faculty of Mathematics and Science, Bogor Agricultural University, Indonesia.

2 Department of Systematic Botany, Institute of Plant Sciences, University of Göttingen, 37073 Göttingen, Germany; corresponding author e-mail: sgradst@uni-goettingen.de

${ }^{3}$ Herbarium Celebense, Tadulako University, Palu, Indonesia.

4 The Herbarium, Singapore Botanic Gardens, 1 Cluny Road, Singapore 259569.
}

Acknowledgements We express our gratitude to Dr. Jochen Heinrichs for help with identification of Plagiochila species and the German Research Foundation (DFG) for financial support.

\section{REFERENCES}

Ariyanti NS, Gradstein SR. 2007. Wallace's line and the distribution of the liverworts of Sulawesi. Cryptogamie, Bryologie 28: 3-14.

Eggers J. 2006. New bryophyte taxon records from tropical countries. Tropical Bryology 27: 107-111.

Gradstein SR, Tan BC, King C, Zhu RL, Drubert C, Pitopang R. 2005. A catalogue of the bryophytes of Sulawesi, Indonesia. Journal of the Hattori Botanical Laboratory 98: 213-257.

He-Nygrén X, Piippo S. 2003. Phylogenetic relationships of the generic complex Chiloscyphus-Lophocolea-Heteroscyphus (Geocalycaceae, Hepaticae): Insights from three chloroplast genes and morphology. Annales Botanici Fennici 40: 317-329.

Hentschel J, Wilson R, Burghardt M, Zündorf H-J, Schneider H, Heinrichs J. 2006. Reinstatement of Lophocoleaceae (Jungermanniopsida) based on chloroplast gene rbcL data: exploring the importance of female involucres for the systematics of Jungermanniales. Plant Systematics and Evolution 258: 211-226.

Piippo S. 1985. Bryophyte flora of the Huon Peninsula, Papua New Guinea. XII. Geocalycaceae. Acta Botanica Fennica 131: 129-167.

Tscharntke T, Leuschner C, Zeller M, Guhardja E, Bidin A(eds). 2007. Stability of tropical rainforest margins, linking ecological, economic and social constrains of land use and conservation. Springer, Berlin. 
Appendix List of bryophyte species newly recorded from Sulawesi since 2005

ANTHOCEROTOPHYTA (hornworts)

Notothylas sp. - Toro: 850-900 m, Ariyanti 227

\section{MARChANTIOPHYTA (liverworts)}

Acrolejeunea arcuata (Nees) Grolle \& Gradst. - Mt Rorekatimbu: 1900 m, Gradstein 12053

Acrolejeunea pycnoclada (Taylor) Schiffn. - Toro: 800-1100 m, Ariyanti 96

Acromastigum divaricatum (Gottsche, Lindenb. \& Nees)A. Evans - Bariri: 1400 m, Gradstein 12022

Anastrophyllum bidens (Reinw., Blume \& Nees) Steph. - Mt Rorekatimbu: 2000 m, Gradstein 12067

Aneura maxima (Schiffn.) Steph. - Bariri: 1300 m, Gradstein 2003 - Mt Rorekatimbu: 1700-2300 m, Gradstein 12045, Angelika 15 - Toro: Ariyanti 181

Anomacaulis flaccidus (Steph.) Grolle - Kalimpaa: 1600 m, Ariyanti 442 - Mt Nokilalaki: 2200-2350 m, Gradstein 11025, Ariyanti 747

Bazzania intermedia (Lindenb. \& Gottsche) Trevis. - Bariri: 1300-1400 m, Gradstein 12093

Calypogeia arguta Mont. \& Nees - Toro: 1000 m, Ariyanti 231

Chandonanthus hirtellus (F.Weber) Mitt. - Bariri: 1400 m, Gradstein 12068 - Mt Nokolalaki: 2350 m, Gradstein 11025

Chandonanthus piliferus Steph. - Kalimpaa: 1600 m, Ariyanti 448 - Mt Nokilalaki: 2000-2350 m, Gradstein 11015, 1102, Ariyanti 738

Cheilolejeunea ceylanica (Gottsche) R.M.Schust. \& Kachroo - Bariri: 1400 m, Gradstein 12027, 12095 - Toro: 900-1100 m, Ariyanti 101, Sporn s.n.

Cheilolejeunea falsinervis (Sande Lac.) R.M.Schust. \& Kachroo - Toro: 1000$1100 \mathrm{~m}$, Ariyanti 102

Cheilolejeunea longiloba (Steph. ex G.Hoffm.) J.J.Engel \& B.C.Tan - Mt Rorekatimbu: 1900-2000 m, Gradstein 12054, 12057

Cheilolejeunea meyeniana (Gottsche, Lindenb. \& Nees) R.M.Schust. \& Kachroo - Toro: 800-1100 m, Ariyanti 104

Cheilolejeunea orientalis (Gottsche) Mizut. - Kalimpaa: 1600 m, Ariyanti 445 - Toro: 800-1100 m, Ariyanti 105

Cheilolejeunea vittata (Steph. ex G.Hoffm.) R.M.Schust. \& Kachroo - Toro: 800-1200 m, Ariyanti 107, Sporn s.n.

Chiloscyphus costatus (Nees) J.J.Engel \& R.M.Schust. (= Lophocolea costata (Nees) Gottsche) - Mt Nokilalaki: 2350 m, Ariyanti 730

Recent molecular-phylogenetic studies have shown that the genus Lophocolea (Dumort.) Dumort. should be merged with Chiloscyphus Corda (He-Nygrén \& Piippo 2003, Hentschel et al. 2006). The latter generic name is the oldest of the two.

Chiloscyphus morobeanus (Piippo) Gradst., comb. nov. (= Lophocolea morobeana Piippo, Acta Bot. Fenn. 131: 160.1985) - Toro: 1000 m, Ariyanti 182 For the transfer of this and other species of Lophocolea to Chiloscyphus see under C. costatus. This species is very close to Chiloscyphus kurzii (Sande Lac.) J.J.Engel \& R.M.Schust. but differs by the presence of trigones (lacking in L. kurzii). Chiloscyphus morobeanus is only known from the type from Papua New Guinea whereas $C$. kurzii is a western Malesian species, being recorded from Java, India and Sri Lanka. Moreover, C. kurzii is a lowland plant, from below $1000 \mathrm{~m}$, whereas C. morobeanus occurs at 1500-1700 m (Piippo 1985).

Chiloscyphus muricatus (Lehm.) J.J.Engel \& R.M.Schust. (= Lophocolea muricata (Lehm.) Nees) - Bariri: 1400 m, Gradstein 12025 - Toro: 1000-1200 m, Ariyanti 140

Cololejeunea dozyana (Sande Lac.) Schiffn. - Mt Rorekatimbu: 1950 m, Eggers (2006)

Cololejeunea equialbi Tixier - Mt Rorekatimbu: 1950 m, Eggers (2006)

Cololejeunea gottschei (Steph.) Mizut. - Kamarora: 700 m, Eggers (2006)

Cololejeunea inflectens (Mitt.) Benedix - Mt Rorekatimbu: 1700-1900 m, Eggers (2006)

Cololejeunea lanciloba Steph. - Kamarora: 700 m, Eggers (2006) - Toro: 800-1100 m, Sporn s.n.

Cololejeunea metzgeriopsis (K.I.Goebel) Gradst., Wilson, Ilkiu-Borges \& Heinrichs (= Metzgeriopsis pusilla K.I.Goebel) - Mt Rorekatimbu: 1950 m, Eggers (2006)

Cololejeunea planissima (Mitt.) Abeyw. - Toro: 1000 m, Ariyanti 108

Cololejeunea schmidtii Steph. - Mt Rorekatimbu: 1950 m, Eggers (2006)

Cololejeunea spinosa (Horik.) Pandé \& R.N.Misra - Toro: 800-1200 m, Ariyanti 109

Colura ari (Steph.) Steph. - Kamarora: 700 m, Eggers (2006)

Colura cf. meyeri Jovet-Ast - Mt Rorekatimbu: 1700 m, Eggers (2006)

Colura tenuicornis (A.Evans) Steph. - Mt Rorekatimbu: 1700 m, Eggers (2006)

Dendrolejeunea fruticosa (Lindenb. \& Gottsche) Lacout. - Toro: 1000-1100 m, Ariyanti 110

Diplasiolejeunea cavifolia (Steph.) Steph. - Toro: 800 m, Sporn s.n.

Drepanolejeunea ternatensis (Gottsche) Steph. - Mt Nokilalaki: 2000 m, Gradstein

11033 - Toro: 800-1200 m, Ariyanti 112, Sporn s.n.

Dumortiera hirsuta (Sw.) Nees - Bariri: 1300 m, Gradstein s.n. - Toro: 1000 m, Ariyanti 277

Frullania eymae S.Hatt.- Toro: 800-1200 m, Ariyanti 114, Sporn s.n.

Frullania hampeana Nees - Toro: 840-1200 m, Ariyanti 118
Frullania intermedia (Reinw., Blume \& Nees) Gottsche, Lindenb. \& Nees - Toro: 840-1000 m, Ariyanti 119

Frullania neosheana S. Hatt. - Mt Nokilalaki: 2250 m, Ariyanti 737 - Toro: 1000 m, Ariyanti 120

Frullania reflexistipula Sande Lac. - Toro: 800-1000 m, Ariyanti 122

Herbertus pilifer Schiffn. - Bariri: 1400 m, Gradstein s.n. - Mt Nokilalaki: 1900 2300 m, Gradstein 11010, 11011 - Mt Rorekatimbu: 1800-2000 m, Gradstein s.n

Heteroscyphus argutus (Reinw., Blume \& Nees) Schiffn. - Bariri: 1400 m, Gradstein s.n. - Mt Nokilalaki: 1000 m, Gradstein s.n. - Toro: 800-1200 m, Ariyanti 123

Heteroscyphus aselliformis (Reinw., Blume \& Nees) Schiffn. - Kalimpaa: 1600 m, Ariyanti 441 - Mt Nokilalaki: 1650-2350 m, Gradstein 11003, 11026, Ariyant 718, 740

Heteroscyphus succulentus (Gottsche) Schiffn - Mt Rorekatimbu: 1700-2000 m, Gradstein 12038 - Toro: 1000 m, Ariyanti 125

Heteroscyphus wettsteinii (Schiffn.) Schiffn. - Mt Nokilalaki 1600 m, Ariyanti 702 Heteroscyphus zollingeri (Gottsche) Schiffn. - Bariri: 1400 m, Gradstein 12019 - Mt Nokilalaki: 1600 m, Ariyanti 701 - Toro: 1000-1200 m, Ariyanti 126 Jungermannia hasskarliana (Nees) Mitt. - Mt Rorekatimbu: 2300 m, Angelika 73

Jungermannia tetragona Lindenb. - Toro: 1000 m, Ariyanti 229

Lejeunea discreta Lindenb. - Bariri: 1400 m, Gradstein 12032 - Toro: 800-1030 m, Ariyanti 128

Lejeunea eifrigrii Mizut. - Toro: 1000 m, Ariyanti 129

Lejeunea exilis (Reinw., Blume \& Nees) Grolle - Toro: 800-1000 m, Ariyanti 130, Sporn s.n.

Lejeunea flava (Sw.) Nees - Mt Nokilalaki: 2000-2100 m, Ariyanti 733 - Mt Rorekatimbu: 1600-1950 m, Eggers (2006), Gradstein 12037 - Toro: 800-1100 m, Ariyanti 131, Sporn s.n.

Lejeunea lumbricoides (Nees) Nees - Mt Nokilalaki: 1900-2350 m, Gradstein 11028, 11053, Ariyanti 729, 756

Lejeunea obscura Mitt. - Bariri: 1400 m, Gradstein 12101 - Toro: 800-1200 m, Ariyanti 132

Lejeunea punctiformis Taylor - Toro: 1000-1100 m, Ariyanti 133

Lejeunea sordida (Nees) Nees - Bariri: 1400 m, Gradstein s.n. - Toro: 8001200 m, Ariyanti 134, Sporn s.n.

Lepidolejeunea bidentula (Steph.) R.M.Schust. - Bariri: 1400 m, Gradstein 12036 - Kalimpaa: 1600 m, Ariyanti 456 - Toro: 1000-1200 m, Ariyanti 135, Sporn s.n.

Lepidozia borneensis Steph. - Mt Nokilalaki: 2350 m, Gradstein 11004

Lepidozia cladorrhiza (Reinw., Blume \& Nees) Nees - Mt Nokilalaki: 2200 m, Gradstein 11039, Ariyanti 725, 749

Lepidozia fernandi-muelleri Steph. - Mt Nokilalaki: 2000-2350 m, Ariyanti 758

Lepidozia grandifolia Steph. - Mt Nokilalaki: 1600 m, Ariyanti 449

Lepidozia hampeana Lindenb. - Mt Nokilalaki: 1600 m, Ariyanti 455

Lepidozia lacerifolia Steph. - Mt Nokilalaki: 2100-2350 m, Ariyanti 732, 744

Lepidozia trichodes (Reinw., Blume \& Nees) Nees - Mt Nokilalaki: 2000-2300 m, Gradstein 11005, 11019, Ariyanti 731 - Mt Rorekatimbu: 2450 m, Angelika 50

Lepidozia wallichiana Gottsche - Kalimpaa: 1600 m, Ariyanti 450 - Mt Nokilalaki: 2000 m, Gradstein 11009, Ariyanti 703, 716 - Mt Rorekatimbu: 2470 m, Angelika 47 - Toro: 1000 m, Ariyanti 136

Leptolejeunea balansae Steph. - Kamaroro: 700 m, Eggers (2006)

Leptolejeunea elliptica (Lehm. \& Lindenb.) Schiffn. - Mt Rorekatimbu: 1900 m, Eggers (2006)

Leptolejeunea epiphylla (Mitt.) Steph. - Kamarora: 700 m, Eggers (2006) - Toro: 800-1100 m, Ariyanti 137, Sporn s.n.

Leptolejeunea maculata (Mitt.) Schiffn. - Kamarora: 700 m, Eggers (2006) - Mt Rorekatimbu: 1900 m, Eggers (2006) - Toro: 800-1100 m, Ariyanti 138

Leptolejeunea vitrea (Nees) Schiffn. - Mt Rorekatimbu: 1900 m, Eggers (2006) Lopholejeunea borneensis (Steph.) Verd. - Toro: 1000-1200 m, Ariyanti 141

Lopholejeunea eulopha (Taylor) Schiffn. - Toro: 800-1200 m, Ariyanti 142, Sporn s.n.

Mastigolejeunea virens (Ángstr.) Steph. - Toro: 800-1100 m, Ariyanti 148, Sporn s.n.

Metalejeunea cucullata (Reinw., Blume \& Nees) Grolle - Bariri: 1400 m, Gradstein s.n. - Toro: 800-1100 m, Ariyanti 149, Sporn s.n.

Notoscyphus lutescens (Lehm. \& Lindenb.) Mitt. - Mt Rorekatimbu: 1700-2000 m, Gradstein 12066

Pallavicinia lyellii (Hook.) Carruth. - Bariri: 1300-1400 m, Gradstein 11092 - Toro: 800-1100 m, Ariyanti 235

Plagiochila dendroides (Nees) Lindenb. - Bariri: 1400 m, Gradstein s.n. - Kalimpaa: $1600 \mathrm{~m}$, Ariyanti 451

Plagiochila hampeana Gottsche - Mt Nokilalaki: 1600 m, Gradstein 11035 (det. J. Heinrichs)

Plagiochila longispica Mitt. - Toro: 800-1000 m, Ariyanti 157

Plagiochila mastigophoroides Inoue - Kalimpaa: 1600 m, Ariyanti 444

Plagiochila obtusa Lindenb. - Toro: 800-1100 m, Ariyanti 158 (det. J. Heinrichs) 
Appendix (cont.)

Plagiochila parvifolia Lindenb. - Toro: 800-1000 m, Ariyanti 159 (det. J. Heinrichs)

Plagiochilion braunianum (Nees) S.Hatt. - Mt Nokilalaki: 2350 m, Gradstein 11020, Ariyanti 743

Porella geheebii (Steph.) S.Hatt. - Kalimpaa: 1600 m, Ariyanti 457

Porella javanica (Gottsche ex Steph.) Inoue - Toro: 1000-1200 m, Ariyanti 155

Porella oblongifolia S.Hatt. - Kalimpaa: 1600 m, Ariyanti 458 - Toro: 1100 m, Ariyanti 159

Porella perrottetiana (Mont.) Trevis. - Bariri: 1400 m, Gradstein 12033 - Toro: 1000 m, Ariyanti 166

Psiloclada clandestina Mitt. - Bariri: 1400 m, Gradstein 12020 - Mt Nokilalaki: 2000 m, Gradstein 11008

Pycnolejeunea contigua (Nees) Grolle - Toro: 1030 m, Ariyanti 168

Radula acutiloba Steph. - Toro: 1000 m, Ariyanti 169

Radula anceps Sande Lac. - Toro: 1100 m, Ariyanti 191

Radula falcata Steph. - Toro: 1000 m, Sporn s.n.

Radula gedena Gottsche ex Steph. - Toro: 800-1000 m, Ariyanti 170

Radula javanica Gottsche - Toro: 1000-1200 m, Ariyanti 171, Sporn s.n.

Radula madagascarensis Steph. - Toro: 1000-1030 m, Ariyanti 172

Radula multiflora Gottsche ex Schiffn. - Toro: 800-1200 m, Ariyanti 173

Radula pinnulata Mitt. - Toro: 830 m, Ariyanti 174

Radula retroflexa Taylor - Toro: 800-1100 m, Ariyanti 175

Radula van-zantenii Yamada - Toro: 830 m, Ariyanti 176, Sporn s.n.

Riccardia sp. 1 - Mt Rorekatimbu: 2470 m, Angelika 46

Riccardia sp. 2 - Mt Rorekatimbu: 2530 m, Angelika 38

Riccardia sp. 3 - Mt Rorekatimbu: 1700-1900 m, Gradstein 12039, 12040

Riccardia sp. 4 - Bariri: 1400 m, Gradstein 12043

Riccardia sp. 5 - Bariri: 1400 m, Gradstein 12044

Schistochila doriae (De Not.) Trevis. - Mt Nokilalaki: 1300-2350 m, Gradstein 11002, 11007

Stenolejeunea apiculata (Sande Lac.) R.M. Schust. - Toro: 800-1200 m, Ariyanti 178, Sporn s.n.

Syzygiella ovalifolia Inoue - Mt Nokilalaki: 2200-2350 m, Gradstein 11012, 11025, Ariyanti 735, 751

Syzygiella subintegerrima (Reinw., Blume \& Nees) Spruce - Bariri: 1300 m, Gradstein 11098 - Mt Nokilalaki: 1800 m, Gradstein 1143 - Mt Rorekatimbu: 1700-1800 m, Eggers (2006), Gradstein 12049, 12050

Telaranea major J.J.Engel \& G.L.Sm. - Bariri: 1400 m, Gradstein 12094 - Mt Nokilalaki: 1650-2350 m, Ariyanti 719, Gradstein 11009

Telaranea neesii (Lindenb.) Fulford - Mt Nokilalaki: 1650-2350 m, Ariyanti 710 Trichocolea tomentella (Ehrh.) Dumort. - Bariri: 1400 m, Gradstein s.n. Mt Nokilalaki: 1200-2350 m, Gradstein 11001, 11037, 11047, Ariyanti 750 - Kalimpaa: 1600 m, Ariyanti 437 - Toro: 1000 m, Ariyanti 192

Tuyamaella jackii (Steph.) Tixier - Toro: $1000 \mathrm{~m}$, Sporn s.n.

Tylimanthus saccatus (Hook.) Mitt. - Mt Nokilalaki: 2000 m, Gradstein 11044 . Ariyanti 724, 746 - Mt Rorekatimbu: 1800 m, Gradstein 12048

Wettsteinia inversa (Sande Lac.) Schiffn. - Mt Nokilalaki: 2000-2300 m, Gradstein 11013, 11014, 11034

Zoopsis liukiuensis Horik. - Bariri: 1300-1400 m, Gradstein 12000 - Mt Nokilalaki: 1600 m, Ariyanti 704

\section{BRYOPHYTA (mosses)}

Acroporium macroturgidum Dixon - Mt Nokilalaki: 2000-2350 m, Ariyanti 514, 541, 553, Sporn s.n.

Arthrocormus schimperi Dozy \& Molk. - Toro: 800-1100 m, Ariyanti 9, 263 - Mt Nokilalaki: $1600 \mathrm{~m}$, Ariyanti s $n$.

Barbella trichophora (Mont.) M.Fleisch. (= Barbella enervis (Thwaites \& Mitt.) M.Fleisch.) - Toro: 800-1100 m, Ariyanti 10, 316 - Kalimpaa: 1600 m, Ariyanti 371

Bryohumbertia walkeri (Mitt.) Frahm - Mt Nokilalaki: 2300 m, Ariyanti 538

Bryum apiculatum Schwägr. - Toro: 800-1100 m, Ariyanti 212, 213 - Trail to Lindu lake from Sedaunta: 900-1150 m, Ariyanti 286

Caduciella mariei (Besch.) Enroth (= Pinnatella microptera M.Fleisch.) - Toro: 800-1100 m, Ariyanti 81

Callicostella papillata (Mont.) Mitt. - Toro: 800-1100 m, Ariyanti 321

Calymperes caugiense Besch. - Toro: 800-1100 m, Ariyanti 13

Calyptothecium subcrispulum Broth. - Toro: 800-1100 m, Sporn s.n.

Chaetomitrium acanthocarpum Bosch \& Sande Lac. - Kalimpaa: 1600 m, Ariyanti 375, 392

Chaetomitrium lanceolatum Bosch \& Sande Lac. - Toro: 800-1100 m, Ariyanti 19, Sporn s.n.

Chaetomitrium leptopoma (Schwägr.) Bosch \& Sande Lac. - Toro: 800-1100 m, Ariyanti 20, Sporn s.n.

Chaetomitrium mayartii H.Akiyama \& M. Suleiman - Toro: 800-1100 m, Ariyanti 21 - Kalimpaa: 1600 m, Ariyanti 376

Chaetomitrium papillifolium Bosch \& Sande Lac. - Toro: 800-1100 m, Ariyanti 23, Sporn s.n.
Chaetomitrium setosum Broth. ex Dixon - Toro: 800-1100 m, Ariyanti 24, Sporn s.n

Clastobryum epiphyllum (Renauld \& Cardot) B.C.Tan \& Touw (= Clastobryum papillosum Williams) - Toro: 800-1100 m, Ariyanti 25, Sporn s.n. - Kalimpaa: $1600 \mathrm{~m}$, Ariyanti 397

Cryptogonium phyllogonioides (Sull.) Isov. - Toro: 800-1100 m, Ariyanti 26 - Wera water fall: $150 \mathrm{~m}$, Ariyanti 429

Cyclodictyon blumeanum (Müll.Hal.) Kuntze - Kalimpaa: 1600 m, Ariyanti 377, 396

Dicranella setifera (Mitt.) A.Jaeger - Toro: 800-1100 m, Ariyanti 214, 215 - Trail to Lindu lake from Sedaunta: 900-1150 m, Ariyanti 290

Distichophyllum osterwaldii M.Fleisch. - Mt Nokilalaki: 1650-2350 m, Ariyanti 535

Distichophyllum spathulatum (Dozy \& Molk.) Dozy \& Molk. - Mt Nokilalaki: 1650-2350 m, Ariyanti 539 - Bariri: 1300 m, Gradstein 12001

Distichophyllum tortile Dozy \& Molk. ex Bosch \& Sande Lac. - Mt Nokilalaki: 1650 m, Ariyanti 539

Ectropothecium dealbatum (Hornsch. \& Reinw.) A.Jaeger - Toro: 800-1100 m, Ariyanti 29, 341, 344

Ephemeropsis tjibodensis K.I.Goebel - Mt Rorekatimbu: 1950 m, Eggers (2006)

Eurhynchium asperisetum E.B.Bartram - Kalimpaa: 1600 m, Ariyanti 378, 393

- Mt Nokilalaki: 1650 m, Ariyanti 536

Fissidens crassinervis Sande Lac. - Toro: 800-1100 m, Ariyanti 35, 210, 255

Fissidens gedehensis M.Fleisch. - Toro 850-900 m, Ariyanti 207

Fissidens papillosus Sande Lac. - Toro: 800-1100 m, Ariyanti 37

Floribundaria pseudofloribunda M.Fleisch. - Toro: 800-1100 m, Ariyanti 40

Floribundaria thuidioides M.Fleisch. - Toro: 800-1100 m, Ariyanti 41

Garckea comosa (Dozy \& Molk.) Wijk \& Margad. - Toro: 800-1100 m, Ariyanti 219, 202

Garovaglia luzonensis Williams - Toro: 800-1100 m, Sporn s.n.

Hyophila involuta (Hook.) A.Jaeger - Wera waterfalls: $250 \mathrm{~m}$, Ariyanti 427

Hypnodendron vitiense Mitt. - Kalimpaa: 1600 m, Ariyanti 404 - Mt Nokilalaki:

1650-2350 m, Ariyanti 573

Hypopterygium aristatum Bosch \& Sande Lac. - Toro: 800-1100 m, Ariyanti 49 Isocladiella sulcularis (Dixon) B.C.Tan \& Mohamed - Toro: 800-1100 m, Ariyanti 51, Sporn s.n.

Jaegerina luzonensis Broth. - Toro: 800-1100 m, Ariyanti 52

Leucophanes massartii Renauld \& Cardot - Toro: 800-1100 m, Ariyanti 57

Leucophanes octoblepharoides Brid. - Toro: 800-1100 m, Ariyanti 58, Sporn s.n.

- Trail to Lindu lake from Sedaunta: 900-1150 m, Ariyanti 296

Macrohymenium strictum Bosch \& Sande Lac. - Mt Nokilalaki: 1650-2100 m,

Ariyanti 561, 545

Macromitrium norrisianum Vitt - Kalimpaa: 1600 m, Ariyanti 345

Macromitrium semipellucidum Dozy \& Molk. - Toro: 800-1100 m, Ariyanti 61

Microdus miquelianus (Mont.) Besch. - Toro: 800-1100 m, Ariyanti 216

Octoblepharum albidum Hedw. - Toro: 800-1100 m, Ariyanti 72, 263-Kalimpaa:

$1600 \mathrm{~m}$, Ariyanti 363, 367

Orthomnion dilatatum (Mitt.) P.C.Chen - Toro: 800-1100 m, Ariyanti 73, 361, Sporn s.n.

Palamocladium nilgheriense (Mont.) Müll.Hal. (= Pleuropus luzonensis Broth.) - Toro: 800-1100 m, Sporn s.n.

Papillaria flexicaulis (Williams) A.Jaeger - Trail to Lindu lake from Sedaunta: 900-1150 m, Ariyanti 392

Philonotis mollis (Dozy \& Molk.) Mitt. - Toro: 850-900 m, Ariyanti 211 - Trail to Lindu lake from Sedaunta: 900-1150 m, Ariyanti 285

Plagiomnium succulentum (Mitt.) T.J.Kop. - Toro: 800-1100 m, Ariyanti 342

Pogonatum piliferum (Dozy \& Molk.) Touw - Toro: 800-1100 m, Ariyanti s.n.

Pseudotaxiphyllum pohliaecarpum (Sull. \& Lesq.) Z.Iwats. - Mt Nokilalaki: 1600 m, Ariyanti 527

Racopilum schmidii (Müll.Hal.) Mitt. - Toro: 800-1100 m, Ariyanti 84 - Trail to Lindu lake from Sedaunta: 900-1150 m, Ariyanti 288

Rhizogonium graffeanum (Müll.Hal.) A.Jaeger - Mt Nokilalaki: 1650-2350 m, Ariyanti 629

Symphysodontella cylindracea (Mont.) M.Fleisch. - Bariri: 1400 m, Gradstein 12016 - Toro: 800-1100 m, Ariyanti 86

Syrrhopodon parasiticus (Brid.) Besch. - Toro: 800-1000 m, Sporn s.n.

Syrrhopodon trachyphyllus Mont. - Toro: 850-900 m, Ariyanti 266

Taxithelium nepalense (Schwägr.) Broth. - Toro: 800-1100 m, Ariyanti 90

Thuidium assimile (Mitt.) A.Jaeger - Toro: 800-1100 m, Ariyanti 91 - Kalimpaa: $1600 \mathrm{~m}$, Ariyanti 351

Trematodon longicollis Michx. - Toro: 800-1100 m, Ariyanti 203 - Kalimpaa: $1600 \mathrm{~m}$, Ariyanti 400

Trismegistia calderensis (Sull.) Broth. - Kalimpaa: 1600 m, Ariyanti 359, 405

Weissia controversa Hedw. - Toro: 850-900 m, Ariyanti 217, 218 